\title{
Cascade Boltzmann - Langevin approach to higher-order current correlations in diffusive metal contacts
}

\author{
K. E. Nagaev \\ Institute of Radioengineering and Electronics, Russian Academy of Sciences, Mokhovaya ulica 11, 101999 Moscow, Russia
}

(October 27, 2018)

\begin{abstract}
The Boltzmann - Langevin approach is extended to calculations of third and fourth cumulants of current in diffusive-metal contacts. These cumulants result from indirect correlations between current fluctuations, which may be considered as "noise of noise". The calculated third cumulant coincides exactly with its quantum-mechanical value. The fourth cumulant tends to its quantummechanical value $-e^{3} I / 105$ at high voltages and to a positive value $2 e^{2} T / 3 R$ at $V=0$ changing its sign at $e V \sim 20 T$.
\end{abstract}

The fluctuation - dissipation theorem relates the spectral density of noise in equilibrium with the corresponding linear response of the system. This is why the second cumulant of current presents new information about the system only under nonequilibrium conditions. Nonequilibrium noise in mesoscopicsystems has been extensively studied in the past decade.1 Unlike the second cumulant of current, its higher cumulants are not related with any average characteristics of the system even in equilibrium. Hence these quantities may be also of experimental interest.

In recent years, higher-order correlations of currents in mesoscopic systems received considerable attention of theorists. This work was pioneered by Levitov and Lesovik 3 who calculated the distribution of charge transmitted through a single-channel quantum contact. Subsequently, these calculations were extended to phase-coherent diffusive contacts in the zero-temperature limit.3 Very recently, the third cumulant of current was calculated for quasi-one-dimensional diffusive-metal contacts for arbitrary temperatures and voltagest using the nonlinear $\sigma$-model and Keldysh formalism 5

Along with the fully quantum-mechanical approach, higher-order correlations have been studied using a semiclassical description, which ignores quantum interference of wave functions yet takes into account the Fermi statistics of electrons. Semiclassical calculations of higher-order cumulants were performed for double-barrier structures 6 using a master equation and for chaotif cavities based on the minimal correlation approach.

A semiclassical Boltzmann - Langevin approach 9 has been very successful in describje the second cumulant of current in diffusive contacts.10 In particular, it gives the same value of the shot noise $S_{I}=2 e I / 3$ as a fully quantum-mechanical treatment 11 This is not surprising because the corrections to semiclassical values from quantum-interference effects like weak localization and mesoscopic fluctuations of gonductance in dirty metals contain a small parameter $121 / p_{F} l_{i m p}$, and the absence of this parameter in the noise magnitude implies that quantum interference is irrelevant to shot noise. As the values of higher cumulants obtained by Lee et al 3 ei- ther do not contain the quantum-interference prefactor as compared to the second cumulant, they can be also obtained semiclassically. This conclusion is supported by recent numerical results, 13 which were obtained in a semiclassical model with exclusion principle.

The present paper extends the semiclassical Boltzmann - Langevin approach to calculations of the third and fourth cumulants of current for diffusive-metal contacts. We show that the main contribution to these quantities still results from second-order correlation functions of the Langevin sources in the Boltzmann - Langevin equation, whereas the effect of higher-order correlations of these sources is negligibly small in the diffusive case. As the result involves several hierarchically coupled second-order correlators, this approach may be termed "cascade".

The Boltzmann-Langevin equation for fluctuations reads

$$
\begin{gathered}
{\left[\frac{\partial}{\partial t}+\mathbf{v} \frac{\partial}{\partial \mathbf{r}}+e \mathbf{E v} \frac{\partial}{\partial \varepsilon}\right] \delta f(\mathbf{p}, \mathbf{r}, t)+\delta I} \\
=-e \delta \mathbf{E} \mathbf{v} \frac{\partial f}{\partial \varepsilon}+\delta J^{e x t}
\end{gathered}
$$

where $\delta I$ is the linearized collision integral and $\delta J^{e x t}$ is the random extraneous flux. Fluctuations of physical quantities are expressed in terms of the fluctuations of the distribution function $\delta f$. For example, the fluctuation of current density is given by an expression

$$
\delta \mathbf{j}(\mathbf{r}, t)=e \sum_{\mathbf{p}} \mathbf{v} \delta f(\mathbf{p}, \mathbf{r}, t)
$$

In principle, the $n$ th-order correlation function of any physical quantity may be calculated provided that the correlation functions of the extraneous sources $\delta J^{e x t}$ are known up to $n$th order

Kogan and Shulmane calculated the second-order correlation function of the extraneous sources based on very simple physical considerations. The collision integral in the Boltzmann equation is of the form

$$
I(\mathbf{p}, \mathbf{r}, t)=\sum_{\mathbf{p}^{\prime}}\left[J\left(\mathbf{p} \rightarrow \mathbf{p}^{\prime}\right)-J\left(\mathbf{p}^{\prime} \rightarrow \mathbf{p}\right)\right],
$$


where $J\left(\mathbf{p} \rightarrow \mathbf{p}^{\prime}\right)$ and $J\left(\mathbf{p}^{\prime} \rightarrow \mathbf{p}\right)$ are the outgoing and incoming scattering fluxes from and to state $\mathbf{p}$. It is natural to assume that all scattering events are local in space and the scattering fluxes between different pairs of states are statistically independent. Furthermore, since scattering events at different instants of time are independent, the scattering between each pair of states presents a Poissonian process whose second-order correlation function $\left\langle\delta J\left(t_{1}\right) \delta J\left(t_{2}\right)\right\rangle$ is proportional to its average rate

$$
J\left(\mathbf{p} \rightarrow \mathbf{p}^{\prime}\right)=W\left(\mathbf{p}, \mathbf{p}^{\prime}\right) f(\mathbf{p}, \mathbf{r}, t)\left[1-f\left(\mathbf{p}^{\prime}, \mathbf{r}, t\right)\right],
$$

where $W\left(\mathbf{p}, \mathbf{p}^{\prime}\right)$ is the classical scattering probability and the factor $1-f$ takes into account the Pauli principle. Hence the correlation function of extraneous sources related with randomness of scattering may be written in a form 9

$$
\begin{gathered}
\left\langle\delta J^{e x t}\left(\mathbf{p}_{1}, \mathbf{r}_{1}, t_{1}\right) \delta J^{e x t}\left(\mathbf{p}_{2}, \mathbf{r}_{2}, t_{2}\right)\right\rangle=\delta\left(\mathbf{r}_{1}-\mathbf{r}_{2}\right) \delta\left(t_{1}-t_{2}\right) \\
\times\left\{\delta_{\mathbf{p}_{1} \mathbf{p}_{2}} \sum_{\mathbf{p}^{\prime}}\left[J\left(\mathbf{p}_{1} \rightarrow \mathbf{p}^{\prime}\right)+J\left(\mathbf{p}^{\prime} \rightarrow \mathbf{p}_{1}\right)\right]-J\left(\mathbf{p}_{1} \rightarrow \mathbf{p}_{2}\right)\right. \\
\left.-J\left(\mathbf{p}_{2} \rightarrow \mathbf{p}_{1}\right)\right\} .
\end{gathered}
$$

The correlation function is positive if $\mathbf{p}_{1}=\mathbf{p}_{2}$ and negative if $\mathbf{p}_{1} \neq \mathbf{p}_{2}$. In the latter case the only correlation is possible through scattering from $\mathbf{p}_{1}$ to $\mathbf{p}_{2}$ and vice versa, but this results in fluctuations of the corresponding occupancies of opposite signs.

In the case of a diffusive metal and low frequencies, the Boltzmann - Langevin scheme is greatly simplified. It is convenient to introduce a fluctuation fo electric potential $\delta \phi$ and random extraneous current 4,15

$$
\delta \mathbf{j}^{e x t}(\mathbf{r}, t)=e \tau \sum_{\mathbf{p}} \mathbf{v} \delta J^{e x t}(\mathbf{p}),
$$

where $\tau$ is the elastic scattering time. The fluctuation of current density is given by an expression

$$
\delta \mathbf{j}=-\sigma \nabla \delta \phi+\delta \mathbf{j}^{e x t},
$$

where $\sigma$ is the metal conductivity. Because pile-up of charge is forbidden in the quasi-static limit, one easily obtains from the condition of current conservation that

$$
\sigma \nabla^{2} \delta \phi=\nabla \mathbf{j}^{e x t}
$$

The correlation function of extraneous currents

$$
\begin{gathered}
\left\langle\delta j_{\alpha}^{e x t}\left(\mathbf{r}_{1}, t_{1}\right)\right. \\
\left.\delta j_{\beta}^{e x t}\left(\mathbf{r}_{2}, t_{2}\right)\right\rangle=2 \sigma \delta_{\alpha \beta} \delta\left(\mathbf{r}_{1}-\mathbf{r}_{2}\right) \delta\left(t_{1}-t_{2}\right) \\
\times \int d \varepsilon f\left(\varepsilon, \mathbf{r}_{1}\right)\left[1-f\left(\varepsilon, \mathbf{r}_{1}\right)\right]
\end{gathered}
$$

is easily obtained from Eq. (5). Expressions (7) - (9) form a complete set of equations for calculating the second cumulant of current.

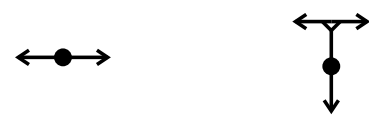

a

b
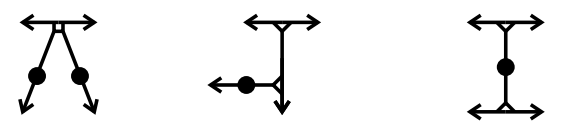

C

FIG. 1. Typical contributions to (a) second, (b) third, and (c) fourth cumulants of current in a diffusive metal. The arrows correspond to current fluctuations at different instances of time. Black circles correspond to the correlators of Langevin sources, empty triangles and squares, to their first and second functional derivatives.

It is natural to use the same notion of independent scattering events for determining the correlation functions of Langevin sources of higher order. According to the ideas of Kogan and Shulman, the Langevin source is a fluctuation of the difference between the outgoing and incoming scattering fluxes into a given state $(\mathbf{p}, \mathbf{r})$. Since each scattering flux between a pair of states is assumed to be a Poissonian process, all its cumulants are proportional to the average scattering rate $1 / \tau$. Hence the third- and fourth-order correlation functions of extraneous currents (6) are proportional to $\tau^{3}$ (see Appendix for details). As will be shown below, the contribution from these correlations is negligibly small as compared to another mechanism related with second-order correlations of Langevin sources. These correlations may be called indirect, or "cascade". The point is that the second-order correlation function of extraneous sources is a functional of the distribution function $f$ and hence may also fluctuate as the current itself. One may roughly imagine these fluctuations as fluctuations of Nyquist noise of a resistor caused by fluctuations of its temperature. A fluctuation $\delta f$ results in a fluctuation

$$
\delta\left\langle\delta j_{\alpha}^{e x t}\left(\mathbf{r}_{1}, t_{1}\right) \delta j_{\beta}^{e x t}\left(\mathbf{r}_{2}, t_{2}\right)\right\rangle=2 \sigma \delta_{\alpha \beta} \delta\left(\mathbf{r}_{1}-\mathbf{r}_{2}\right) \delta\left(t_{1}-t_{2}\right)
$$

$$
\times \int d \varepsilon\left[1-2 f\left(\varepsilon, \mathbf{r}_{1}\right)\right] \delta f\left(\varepsilon, \mathbf{r}_{1}, t_{1}\right)
$$

Note that the time scale for the fluctuation $\delta f$ is set by a characteristic relaxation time and hence they are slow as compared to the duration of a scattering event. As $\delta f$ is also correlated with fluctuations of other quantities, this "noise of noise" results in higher-order correlations 
of currents. Hence even Gaussian extraneous sources may produce non-Gaussian fluctuations.

In a symbolic form, the expression for indirect thirdorder correlations may be written in a form

$$
\left\langle\delta I_{1} \delta I_{2} \delta I_{3}\right\rangle=P_{123}\left\{\frac{\delta\left\langle\delta I_{1} \delta I_{2}\right\rangle}{\delta f_{4}}\left\langle\delta f_{4} \delta I_{3}\right\rangle\right\},
$$

where $P_{123}$ denotes a summation over all inequivalent permutations of indices (123) and $\delta\langle\ldots\rangle / \delta f_{4}$ denotes a functional derivative with respect to $f\left(\varepsilon_{4}, \mathbf{r}_{4}, t_{4}\right)$. The products imply a convolution over the arguments of the distribution functions with repeating indices. The irreducible fourth-order correlation function is presented by three groups of terms

$$
\begin{gathered}
\left\langle: \delta I_{1} \delta I_{2} \delta I_{3} \delta I_{4}:\right\rangle=P_{1234}\left\{\frac{\delta^{2}\left\langle\delta I_{1} \delta I_{2}\right\rangle}{\delta f_{5} \delta f_{6}}\left\langle\delta f_{5} \delta I_{3}\right\rangle\left\langle\delta f_{6} \delta I_{4}\right\rangle\right. \\
+\frac{\delta\left\langle\delta I_{1} \delta I_{2}\right\rangle}{\delta f_{5}} \frac{\delta\left\langle\delta f_{5} \delta I_{3}\right\rangle}{\delta f_{6}}\left\langle\delta f_{6} \delta I_{4}\right\rangle \\
\left.+\frac{\delta\left\langle\delta I_{1} \delta I_{2}\right\rangle}{\delta f_{5}}\left\langle\delta f_{5} \delta f_{6}\right\rangle \frac{\delta\left\langle\delta I_{3} \delta I_{4}\right\rangle}{\delta f_{6}}\right\}
\end{gathered}
$$

The corresponding contributions are schematically illustrated by diagrams in Fig. 1.

To evaluate expressions (10) and (11), one must know the second-order correlation function of fluctuations $\delta f$. In the quasi-static limit, the Boltzmann - Langevin equation may be easily transformed into a stochastic diffusion equation for the fluctuation $\delta f(\varepsilon, \mathbf{r}, t)$

$$
D \nabla^{2} \delta f(\varepsilon, \mathbf{r})=\nabla \delta \mathbf{F}^{e x t},
$$

where

$$
\delta \mathbf{F}^{e x t}(\varepsilon)=\frac{\tau}{N_{F}} \sum_{\mathbf{p}} \mathbf{v} \delta J^{e x t}(\mathbf{p}) \delta\left(\varepsilon_{\mathbf{p}}-\varepsilon\right),
$$

so one obtains from (5) that

$$
\begin{aligned}
&\left\langle\delta F_{\alpha}^{e x t}(\varepsilon, \mathbf{r}) \delta F_{\beta}^{e x t}\left(\varepsilon^{\prime}, \mathbf{r}^{\prime}\right)\right\rangle=2 \frac{D}{N_{F}} \delta\left(\mathbf{r}-\mathbf{r}^{\prime}\right) \delta\left(t-t^{\prime}\right) \\
& \delta\left(\varepsilon-\varepsilon^{\prime}\right) \delta_{\alpha \beta} f(\varepsilon)[1-f(\varepsilon)] \\
&\left\langle\delta F_{\alpha}^{e x t}(\varepsilon, \mathbf{r}) \delta j_{\beta}^{e x t}\left(\mathbf{r}^{\prime}\right)\right\rangle=2 e D \delta\left(\mathbf{r}-\mathbf{r}^{\prime}\right) \delta\left(t-t^{\prime}\right) \\
& \times \delta_{\alpha \beta} f(\varepsilon)[1-f(\varepsilon)],
\end{aligned}
$$

where $D$ is the diffusion coefficient and $N_{F}$ is the Fermi density of states.

Consider the case of a quasi-one-dimensional diffusive wire of length $L$ connecting two massive electrodes with a voltage drop $V$ across it. In this case, all quantities may be considered as depending only on the longitudinal coordinate $x$ along the wire. The solution of Eq. (12) is of the form

$$
\begin{gathered}
\delta f(x)=\frac{1}{D S_{0}} \int_{0}^{L} d x^{\prime} K\left(x, x^{\prime}\right) \int d^{2} r_{\perp} \delta F_{x}^{e x t}\left(\varepsilon, x, \mathbf{r}_{\perp}\right), \\
K\left(x, x^{\prime}\right)=\theta\left(x-x^{\prime}\right)-x / L
\end{gathered}
$$

where $S_{0}$ is the area of the cross-section of the wire and $\mathbf{r}_{\perp}$ are the transverse coordinates. Because the pile-up of charge in the contact is forbidden in the quasi-static limit, the fluctuation of current is independent of $x$ and is obtained by ayeraging the extraneous current over the contact volume 10

$$
\delta I=\int_{0}^{L} \frac{d x}{L} \int d^{2} r_{\perp} \delta j_{x}^{e x t} .
$$

The low-frequency Fourier transform of the correlation function of $\delta I\left(t_{1}\right)$ and $\delta I\left(t_{2}\right)$ is given by

$$
\begin{gathered}
\left\langle\delta I\left(\omega_{1}\right) \delta I\left(\omega_{2}\right)\right\rangle=\delta\left(\omega_{1}+\omega_{2}\right) \\
\times \frac{4 \pi}{R L} \int_{0}^{L} d x \int d \varepsilon f(\varepsilon, x)[1-f(\varepsilon, x)],
\end{gathered}
$$

where $R$ is the resistance of the contact. By giving the distribution function a variance $\delta f(\varepsilon, x)$, one easily obtains the functional derivative of Eq. (18) with respect to $f\left(\varepsilon, x, \omega_{3}\right)$ in the form

$$
\frac{\delta\left\langle\delta I\left(\omega_{1}\right) \delta I\left(\omega_{2}\right)\right\rangle}{\delta f\left(\varepsilon, x, \omega_{3}\right)}=\delta\left(\omega_{1}+\omega_{2}-\omega_{3}\right) \frac{4 \pi}{R L}[1-2 f(\varepsilon, x)] .
$$

The second functional derivative of the same quantity is just

$$
\begin{gathered}
\frac{\delta^{2}\left\langle\delta I\left(\omega_{1}\right) \delta I\left(\omega_{2}\right)\right\rangle}{\delta f\left(\varepsilon_{3}, x_{3}, \omega_{3}\right) \delta f\left(\varepsilon_{4}, x_{4}, \omega_{4}\right)}=-\delta\left(\omega_{1}+\omega_{2}-\omega_{3}-\omega_{4}\right) \\
\times \delta\left(\varepsilon_{3}-\varepsilon_{4}\right) \delta\left(x_{3}-x_{4}\right) \frac{8 \pi}{R L} .
\end{gathered}
$$

The right-hand side of Eq. (10) allows three inequivalent permutations of indices. Hence the low-frequency Fourier transform of the third cumulant of current

$$
\begin{gathered}
S_{3}\left(\omega_{1}, \omega_{2}\right)=\int d\left(t_{1}-t_{3}\right) \int d\left(t_{2}-t_{3}\right) \\
\times \exp \left[i \omega_{1}\left(t_{1}-t_{3}\right)+i \omega_{2}\left(t_{2}-t_{3}\right)\right]\left\langle\delta I\left(t_{1}\right) \delta I\left(t_{2}\right) \delta I\left(t_{3}\right)\right\rangle
\end{gathered}
$$

is given by an expression

$$
\begin{gathered}
S_{3}(0,0)=12 \frac{e}{R L^{2}} \int d \varepsilon \int d x \int d x^{\prime}[1-2 f(\varepsilon, x)] K\left(x, x^{\prime}\right) \\
\times f\left(\varepsilon, x^{\prime}\right)\left[1-f\left(\varepsilon, x^{\prime}\right)\right] .
\end{gathered}
$$


The first, second, and the third terms in the right-hand side of Eq. (11) allow 6, 12, and 3 inequivalent permutations of indices, respectively. Hence the low-frequency Fourier transform of fourth cumulant of current

$$
\begin{gathered}
S_{4}\left(\omega_{1}, \omega_{2}, \omega_{3}\right)=\int d\left(t_{1}-t_{4}\right) \int d\left(t_{2}-t_{4}\right) \int d\left(t_{3}-t_{4}\right) \\
\times \exp \left[i \omega_{1}\left(t_{1}-t_{4}\right)+i \omega_{2}\left(t_{2}-t_{4}\right)+i \omega_{3}\left(t_{3}-t_{4}\right)\right] \\
\times\left\langle: \delta I\left(t_{1}\right) \delta I\left(t_{2}\right) \delta I\left(t_{3}\right) \delta I\left(t_{4}\right):\right\rangle
\end{gathered}
$$

may be written in a form

$$
S_{4}(0,0,0)=6 S_{4-1}+12 S_{4-2}+3 S_{4-3},
$$

where

$$
\begin{gathered}
S_{4-1}=-16 \frac{e^{2}}{R L^{3}} \int d \varepsilon \int_{0}^{L} d x \int_{0}^{L} d x_{1} K\left(x, x_{1}\right) \\
\times f\left(\varepsilon, x_{1}\right)\left[1-f\left(\varepsilon, x_{1}\right)\right] \\
\times \int_{0}^{L} d x_{2} K\left(x, x_{2}\right) f\left(\varepsilon, x_{2}\right)\left[1-f\left(\varepsilon, x_{2}\right)\right], \\
S_{4-2}^{L} 8 \frac{e^{2}}{R L^{3}} \int d \varepsilon \int_{0}^{L} d x[1-2 f(\varepsilon, x)] \\
\times \int_{0}^{L} d x_{1} K\left(x, x_{1}\right)\left[1-2 f\left(\varepsilon, x_{1}\right)\right] \\
\times \int_{0}^{L} d x_{2} K\left(x, x_{2}\right) f\left(\varepsilon, x_{2}\right)\left[1-f\left(\varepsilon, x_{2}\right)\right],
\end{gathered}
$$

and

$$
\begin{aligned}
& S_{4-3}=8 \frac{e^{2}}{R L^{3}} \int d \varepsilon \int_{0}^{L} d x_{1}\left[1-2 f\left(\varepsilon, x_{1}\right)\right] \\
& \times \int_{0}^{L} d x_{2}\left[1-2 f\left(\varepsilon, x_{2}\right)\right] \\
& \times \int_{0}^{L} d x K\left(x_{1}, x\right) K\left(x_{2}, x\right) f(\varepsilon, x)[1-f(\varepsilon, x)] .
\end{aligned}
$$

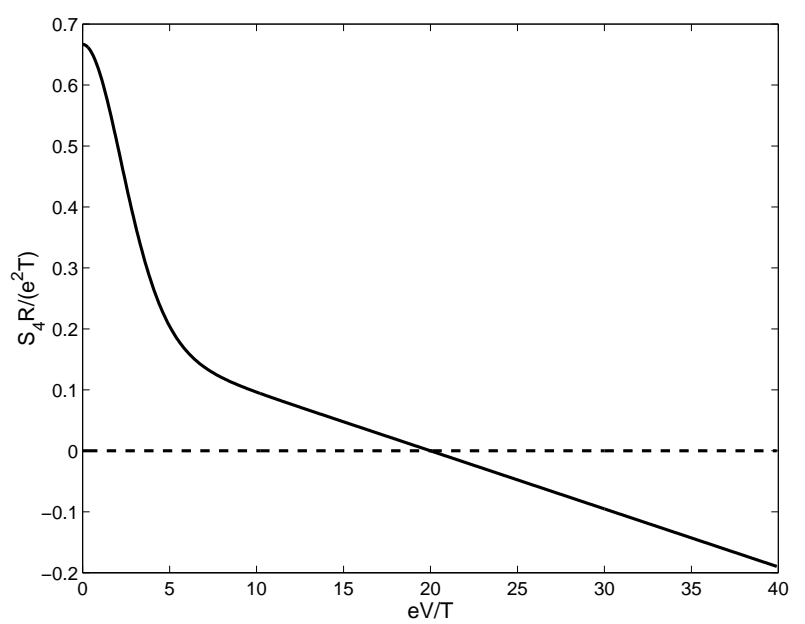

FIG. 2. Voltage dependence of the fourth cumulant of current for a long diffusive contact

The distribution function inside the contact is given by an expression 10

$$
f(\varepsilon, x)=\left(1-\frac{x}{L}\right) f_{0}(\varepsilon+e V / 2)+\frac{x}{L} f_{0}(\varepsilon-e V / 2),
$$

where $f_{0}(\varepsilon)$ is the Fermi distribution function. The integration in Eqs. (21) - (25) is easily performed. One obtains that the third cumulant is equal

$$
S_{3}=\frac{1}{15} \frac{e}{R} \frac{e V \cosh (e V / T)+12 T \sinh (e V / T)-13 e V}{\cosh (e V / T)-1} .
$$

This is essentially the sqme expression that was obtained by Gutman and Gefent using the nonlinear $\sigma$-model. It gives

$$
S_{3}=\frac{1}{15} \frac{e^{2} V}{R}
$$

at high voltages in agreement with Lee et al $\mathbf{B}^{3}$ and

$$
S_{3}=\frac{1}{3} \frac{e^{2} V}{R}
$$

in the low-voltage or high-temperature limit.

The fourth cumulant of current is given by an expression

$$
\begin{gathered}
S_{4}=-\frac{1}{420} \frac{e^{2}}{R} \frac{1}{\sinh ^{3}(e V / 2 T)}\left[e V \cosh \left(\frac{3 e V}{2 T}\right)\right. \\
-20 T \sinh \left(\frac{3 e V}{2 T}\right)-313 e V \cosh \left(\frac{e V}{2 T}\right) \\
\left.+684 T \sinh \left(\frac{e V}{2 T}\right)\right] .
\end{gathered}
$$

It gives 


$$
S_{4}=-\frac{1}{105} \frac{e^{3} V}{R}
$$

in the high-voltage limit in agreement with the results of Lee et al. in Eq. (22) is nonzero and

$$
S_{4}=\frac{2}{3} \frac{e^{2} T}{R}
$$

This result seems to be new. The positive sign of $S_{4}$ in equilibrium is in a qualitative agreement with the shape of distribution function of current numerically obtained by Belzig and Nazarov. 16 The behavior of $S_{4}(V)$ is shown in Fig. 2. The fourth cumulant changes its sign at $\mathrm{eV} \approx 20 T$, hence the distribution of current changes from super-Gaussian to sub-Gaussian as the voltage increases.

It has been proposed recently 6 that the third cumulant of noise may be used for determining the effective charge of quasiparticles. In other words, it may give the same information as the shot noise even at low voltages $\mathrm{eV} \ll T$. Since the fourth cumulant is nonzero even at zero voltage, one may obtain a nontrivial information about the system from measurements of equilibrium fluctuations. Measurements of fluctuations of noise power, which are termed "the second spectral density" and are closely related with the Fourier transform of the fourth cumulant 18 were successfully implemented by a number of authors who studied the $1 / f$ noise 1921

In summary, we have shown that higher cumulants of current in diffusive-metal contacts may be described by the semiclassical Boltzmann - Langevin theory. The semiclassical fluctuations appear to be non-Gaussian both in equilibrium and at high voltages 22 The present approach may be also applied to semiclassical nondiffusive systems. In this case, one has to take into account also higher-order local correlators of extraneous sources.

The author acknowledges a helpful discussion with Sh. M. Kogan. This work was supported by Russian Foundation for Basic Research, grant 01-02-17220, and by an INTAS Open grant, and by an INTAS Open grant 20011B-14.

\section{APPENDIX}

The third central moment of $\delta J^{e x t}$ is easily found from the same considerations as the second moment. Much like the second-order correlator, it is local in space. As the collision integral involves only single-particle transitions between pairs of states, the triple correlator $\left\langle\delta J^{e x t}\left(\mathbf{p}_{1}\right) \delta J^{e x t}\left(\mathbf{p}_{2}\right) \delta J^{e x t}\left(\mathbf{p}_{3}\right)\right\rangle$ is nonzero only if at least two of these states coincide. We also make use of the fact that the third-order correlator of a scattering flux from one state to another, which is assumed to be Poissonian, is proportional to the average scattering rate between these states. The sign of the contribution from each scattering process should be determined by multiplying the signs of occupancy changes in states $\mathbf{p}_{1}, \mathbf{p}_{2}$, and $\mathbf{p}_{3}$ when the corresponding scattering event takes place. Hence the correlator may be written in a form

$$
\begin{gathered}
\left\langle\delta J^{\text {ext }}\left(\mathbf{p}_{1}, \mathbf{r}_{1}, t_{1}\right) \delta J^{\text {ext }}\left(\mathbf{p}_{2}, \mathbf{r}_{2}, t_{2}\right) \delta J^{\text {ext }}\left(\mathbf{p}_{3}, \mathbf{r}_{3}, t_{3}\right)\right\rangle \\
=\delta\left(\mathbf{r}_{1}-\mathbf{r}_{2}\right) \delta\left(\mathbf{p}_{2}-\mathbf{r}_{3}\right) \delta\left(t_{1}-t_{2}\right) \delta\left(t_{2}-t_{3}\right) \\
\times\left\{\delta_{\mathbf{p}_{1} \mathbf{p}_{2}} \delta_{\mathbf{p}_{2} \mathbf{p}_{3}} \sum_{\mathbf{p}^{\prime}}\left[J\left(\mathbf{p}^{\prime} \rightarrow \mathbf{p}_{1}\right)-J\left(\mathbf{p}_{1} \rightarrow \mathbf{p}^{\prime}\right)\right]\right. \\
+\delta_{\mathbf{p}_{1} \mathbf{p}_{2}}\left[J\left(\mathbf{p}_{1} \rightarrow \mathbf{p}_{3}\right)-J\left(\mathbf{p}_{3} \rightarrow \mathbf{p}_{1}\right)\right] \\
+\delta_{\mathbf{p}_{2} \mathbf{p}_{3}}\left[J\left(\mathbf{p}_{2} \rightarrow \mathbf{p}_{1}\right)-J\left(\mathbf{p}_{1} \rightarrow \mathbf{p}_{2}\right)\right] \\
\left.+\delta_{\mathbf{p}_{1} \mathbf{p}_{3}}\left[J\left(\mathbf{p}_{1} \rightarrow \mathbf{p}_{2}\right)-J\left(\mathbf{p}_{2} \rightarrow \mathbf{p}_{1}\right)\right]\right\},
\end{gathered}
$$

where the scattering fluxes $J$ are given by Eq. (雨). It is easily verified that this correlator conserves the total number of electrons at a given point.

Consider now the fourth-order irreducible correlator of extraneous sources. The term "irreducible" means that only the part of the correlator that cannot be decoupled into products of second-order correlators is considered. This correlator is zero for a Gaussian noise but is proportional to the average rate for a Poissonian process. Using the same ideas, the irreducible correlator may be written in a form

$$
\begin{aligned}
& \left\langle: \delta J^{e x t}\left(\mathbf{p}_{1}, \mathbf{r}_{1}, t_{1}\right) \delta J^{e x t}\left(\mathbf{p}_{2}, \mathbf{r}_{2}, t_{2}\right) \delta J^{e x t}\left(\mathbf{p}_{3}, \mathbf{r}_{3}, t_{3}\right) \delta J^{\text {ext }}\left(\mathbf{p}_{4}, \mathbf{r}_{4}, t_{4}\right):\right\rangle= \\
& \delta\left(\mathbf{r}_{1}-\mathbf{r}_{2}\right) \delta\left(\mathbf{r}_{2}-\mathbf{r}_{3}\right) \delta\left(\mathbf{r}_{3}-\mathbf{r}_{4}\right) \delta\left(t_{1}-t_{2}\right) \delta\left(t_{2}-t_{3}\right) \delta\left(t_{3}-t_{4}\right) \\
& \times\left\{\delta_{\mathbf{p}_{1} \mathbf{p}_{2}} \delta_{\mathbf{p}_{2} \mathbf{p}_{3}} \delta_{\mathbf{p}_{3} \mathbf{p}_{4}} \sum_{\mathbf{p}^{\prime}}\left[J\left(\mathbf{p}^{\prime} \rightarrow \mathbf{p}_{1}\right)+J\left(\mathbf{p}_{1} \rightarrow \mathbf{p}^{\prime}\right)\right]-\delta_{\mathbf{p}_{1} \mathbf{p}_{2}} \delta_{\mathbf{p}_{2} \mathbf{p}_{3}}\left[J\left(\mathbf{p}_{1} \rightarrow \mathbf{p}_{4}\right)+J\left(\mathbf{p}_{4} \rightarrow \mathbf{p}_{1}\right)\right]\right. \\
& -\delta_{\mathbf{p}_{1} \mathbf{p}_{2}} \delta_{\mathbf{p}_{2} \mathbf{p}_{4}}\left[J\left(\mathbf{p}_{1} \rightarrow \mathbf{p}_{3}\right)+J\left(\mathbf{p}_{3} \rightarrow \mathbf{p}_{1}\right)\right]-\left(\delta_{\mathbf{p}_{1} \mathbf{p}_{3}} \delta_{\mathbf{p}_{3} \mathbf{p}_{4}}+\delta_{\mathbf{p}_{2} \mathbf{p}_{3}} \delta_{\mathbf{p}_{3} \mathbf{p}_{4}}\right)\left[J\left(\mathbf{p}_{1} \rightarrow \mathbf{p}_{2}\right)+J\left(\mathbf{p}_{2} \rightarrow \mathbf{p}_{1}\right)\right]
\end{aligned}
$$




$$
\begin{gathered}
+\delta_{\mathbf{p}_{1} \mathbf{p}_{2}} \delta_{\mathbf{p}_{3} \mathbf{p}_{4}}\left[J\left(\mathbf{p}_{1} \rightarrow \mathbf{p}_{3}\right)+J\left(\mathbf{p}_{3} \rightarrow \mathbf{p}_{1}\right)\right] \\
\left.+\left(\delta_{\mathbf{p}_{1} \mathbf{p}_{3}} \delta_{\mathbf{p}_{2} \mathbf{p}_{4}}+\delta_{\mathbf{p}_{1} \mathbf{p}_{4}} \delta_{\mathbf{p}_{2} \mathbf{p}_{3}}\right)\left[J\left(\mathbf{p}_{1} \rightarrow \mathbf{p}_{2}\right)+J\left(\mathbf{p}_{2} \rightarrow \mathbf{p}_{1}\right)\right]\right\} .
\end{gathered}
$$

In the diffusive limit, it is convenient to pass from extraneous fluxes in the Boltzmann - Langevin equation to extraneous currents in the drift-diffusion equation by means of Eq. (6). The third-order correlator of extraneous currents is of the form

$$
\begin{gathered}
\left\langle\delta j_{\alpha}^{e x t}\left(\mathbf{r}_{1}, t_{1}\right) \delta j_{\beta}^{e x t}\left(\mathbf{r}_{2}, t_{2}\right) \delta j_{\gamma}^{e x t}\left(\mathbf{r}_{3}, t_{3}\right)\right\rangle \\
=\delta\left(\mathbf{r}_{1}-\mathbf{r}_{2}\right) \delta\left(\mathbf{r}_{2}-\mathbf{r}_{3}\right) \delta\left(t_{1}-t_{2}\right) \delta\left(t_{2}-t_{3}\right) \\
\times \tau^{3} e^{3} \sum_{\mathbf{p p ^ { \prime }}}\left(v_{\alpha} v_{\beta} v_{\gamma}-v_{\alpha}^{\prime} v_{\beta} v_{\gamma}-v_{\alpha} v_{\beta}^{\prime} v_{\gamma}-v_{\alpha} v_{\beta} v_{\gamma}^{\prime}\right) \\
\times\left[J\left(\mathbf{p}^{\prime} \rightarrow \mathbf{p}\right)-J\left(\mathbf{p} \rightarrow \mathbf{p}^{\prime}\right)\right] .
\end{gathered}
$$

Though the scattering fluxes $J$ are proportional to $1 / \tau$, the bracketed difference vanishes in equilibrium and is proportional to the anisotropic part of the distribution function in the case of applied bias. As this part is proportional to $\tau$ in the diffusive approximation, Eq. (A3) is at least of the order of $\tau^{3}$.

The fourth-order irreducible correlator of extraneous currents is of the form

$$
\begin{gathered}
\left\langle: \delta j_{\alpha}^{e x t}\left(\mathbf{r}_{1}, t_{1}\right) \delta j_{\beta}^{e x t}\left(\mathbf{r}_{2}, t_{2}\right) \delta j_{\gamma}^{e x t}\left(\mathbf{r}_{3}, t_{3}\right) \delta j_{\delta}^{e x t}\left(\mathbf{r}_{4}, t_{4}\right):\right\rangle \\
=\frac{16}{15} e^{2} l_{i m p}^{2} \sigma \delta\left(\mathbf{r}_{1}-\mathbf{r}_{2}\right) \delta\left(\mathbf{r}_{2}-\mathbf{r}_{3}\right) \delta\left(\mathbf{r}_{3}-\mathbf{r}_{4}\right) \\
\times \delta\left(t_{1}-t_{2}\right) \delta\left(t_{2}-t_{3}\right) \delta\left(t_{3}-t_{4}\right)\left(\delta_{\alpha \beta} \delta_{\gamma \delta}+\delta_{\alpha \gamma} \delta_{\beta \delta}+\delta_{\alpha \delta} \delta_{\beta \gamma}\right) \\
\int d \varepsilon f\left(\varepsilon, \mathbf{r}_{1}\right)\left[1-f\left(\varepsilon, \mathbf{r}_{1}\right)\right],
\end{gathered}
$$

where $\sigma$ is the conductivity. Hence the fourth-order correlator is also of the order $\tau^{3}$.
${ }^{1}$ Y. Blanter and M. Büttiker, Phys. Rep. 336, 1 (2000).

${ }^{2}$ L. S. Levitov and G. B. Lesovik, Pis'ma Zh. Eksp. Teor. Fiz. 58, 225 (1993) [JETP Lett. 58, 230 (1993)].

${ }^{3}$ H. Lee, L. S. Levitov. and A. Yu. Yakovets, Phys. Rev. B 51, 4079 (1995).

${ }^{4}$ D. B. Gutman and Y. Gefen, cond-mat/0201007

${ }^{5}$ A. Kamenev, A. Andreev, Phys. Rev. B 60, 2218 (1999).

${ }^{6}$ M. J. M. de Jong, Phys. Rev. B 54, 8144 (1996).

${ }^{7}$ Ya. M. Blanter, H. Schomerus, and C. W. J. Beenakker, Physica E 11, 1 (2001).

${ }^{8}$ Ya. M. Blanter and E. V. Sukhorukov, Phys. Rev. Lett. 84, 1280 (2000).

${ }^{9}$ Sh. M. Kogan and A. Ya. Shul'man, Zh. Eksp. Teor. Fiz. 56, 862 (1969) [Sov. Phys. JETP 29, 467 (1969)].

${ }^{10}$ K. E. Nagaev, Phys. Lett. A 169, 103 (1992).

${ }^{11}$ C. W. J. Beenakker and M. Büttiker, Phys. Rev. B 46, 1889 (1992).

12 A. A. Abrikosov, Fundamentals of the Theory of Metals, North Holland, Amsterdam, 1988.

${ }^{13}$ P.-E. Roche and B. Douçot, cond-mat/0202149.

${ }^{14}$ K. E. Nagaev, Phys. Rev. B 57, 4628 (1998).

${ }^{15}$ E. V. Sukhorukov and D. Loss, Phys. Rev. Lett. 80, 4959 (1998); Phys. Rev. B 59, 13054 (1999).

${ }^{16}$ W. Belzig and Yu. V. Nazarov, Phys. Rev. Lett. 87, 067006 (2001).

${ }^{17}$ L. S. Levitov and M. Reznikov, cond-mat/0111057.

${ }^{18}$ Sh. Kogan, Electronic Noise and Fluctuations in Solids (Cambridge University Press, Cambridge, 1996).

${ }^{19}$ G. A. Garfunkel, G. B. Alers, and M. B. Weissman, Phys. Rev. B 41, 4901 (1990).

${ }^{20}$ N. E. Israeloff, G. B. Alers, and M. B. Weissman, Phys. Rev. B 44, 12613 (1991).

${ }^{21}$ C. E. Parman, N. E. Israeloff, and J. Kakalios, Phys. Rev. Lett. 69, 1097 (1992).

${ }^{22}$ Both of thesf statements are in contrast with the claim made in Ref. 4 The conclusion of these authors was based on the fact that the third cumulant of current cannot be obtained correctly with purely local extraneous sources in the standard version of the Boltzmann-Langevin approach, which does not involve the above cascade mechanism. However even this standard version yields non-Gaussian fluctuations in the quasi-ballistic regime (see Appendix). 